\title{
Synthesis, structure and magnetic properties of an iron(II) complex with nitronyl nitroxides
}

\author{
Masayuki Nihei, Tadashi Maeshima, Yuka Kose, and Hiroki Oshio* \\ Graduate School of Pure and Applied Sciences, University of Tsukuba, Tennodai 1-1-1,
}

Tsukuba 305-8571, Japan

Keywords: Tridentate nitrogen ligand, nitronyl nitroxide, molecular magnetism, Iron,

spin-crossover.

\begin{abstract}
An iron(II) complex with nitronyl nitroxides, $\left[\mathrm{Fe}^{\mathrm{II}}(\mathrm{dppNN})_{2}\right]\left(\mathrm{BF}_{4}\right)_{2} \cdot \mathrm{CH}_{3} \mathrm{COCH}_{3}$

(dppNN $=$

2,6-di(pyrazol-1-yl)-4-(4,4,5,5-tetramethyl-1-oxido-3-ylooxy-4,5-dihydro-3H -imidazol-2'-yl)pyridine) was synthesized. In $\mathbf{1}$ the central iron(II) ion was coordinated by two tridentate ligands with nitroniyl nitroxides. Magnetic susceptibility measurements showed that $\chi_{\mathrm{m}} T$ values below $130 \mathrm{~K}$ was almost temperature independent, while upon increasing temperature $\chi_{\mathrm{m}} T$ values showed gradual increase, suggesting an occurrence of a spin transition
\end{abstract}


from low to high spin state. Green light irradiation on powder sample at 5 $\mathrm{K}$ resulted in spin conversion (LIESST).

\section{Introduction}

Tridentate N-heterocyclic ligands have been used over the last century as effective and stable complexing agents for transition metal ions. These ligands have been used to build supramolecular coordination polymers[1] and denderimers[2], which might have interesting catalytic, photochemical and redox properties.[3] Nitronyl nitroxides, on the other hand, have been extensively used as building blocks for pure organic ferromagnets[4] and as bridging ligands for paramagnetic metal ions.[5] A number of transition metal complexes with nitronyl nitroxide-functionalized pyridines[6], polypyridines[7], imidazole and pyrazol[8] have been synthesized. Gateschi et al. reported that a so-called single chain magnet of $\left[\mathrm{Co}(\mathrm{hfac})_{2}(\mathrm{rad})\right](\mathrm{hfac}=$ hexafluoroacetylacetonate, $\mathrm{rad}=2$-( $p$-methoxyphenyl)nitronylnitroxide), which showed slow magnetic relaxation of spin flips.[9] 
Tridentate nitrogen ligand 2,6-di(pyrazol-1-yl)pyridine (dpp) and its derivatives, which are considered as analogues of terpyridine, ligate an iron(II) ion and complex molecules showed thermally and light induced spin-crossover, the latter called light induced excited spin state trapping (LIESST) phenomena.[10] An Iron(II) complex of dpp ligands containing nitronyl nitroxide radicals, therefore, can be a good candidates for the hybrid system exhibiting synergy of bulk magnetism and spin transitions. We report here preparations of a new tridentate ligand with the nitronyl nitroxide (Scheme 1) and its iron(II) complex, and the magnetic properties were studied.

\section{Experimental}

\section{2-1. Syntheses}

All reagents were purchased and used without further purification. ethyl 2,6-di(pyrazol-1-yl)isonicotinate[11] and N,N'-dihydroxy-2,3-diamino2,3-dimethylbutane [12] were synthesized according to the literature 
methods.

dipyrazol-3-yl-4-hydroxymethylpyridine (2). Sodium borohydride (1.48

g, $39.2 \mathrm{mmol}$ ) was added to dipyrazol-3-yl-4-carboxyethylpyridine (2.22 g, $7.84 \mathrm{mmol})$ in ethanol $(150 \mathrm{ml})$ and the mixture was refluxed for 6 hours. The resulting mixture was evaporated, and $60 \mathrm{ml}$ of water and $23.3 \mathrm{ml}$ of $1 \mathrm{M}$ $\mathrm{HCl}$ aq. was added to the residue. The crude product was extracted with dichloromethane and purified by recrystallization from dichloromethane/hexane to yield $1.47 \mathrm{~g}$ of 2 (6.09 mmol, $77.7 \%)$. Anal. Calcd for $\mathrm{C}_{12} \mathrm{H}_{11} \mathrm{~N}_{5} \mathrm{O}_{1}$ : C, 59.74; H, 4.60; N, 29.03. Found: C, 59.88; H, 4.66; N, 29.22\%. ${ }^{1} \mathrm{H}-\mathrm{NMR}\left(270 \mathrm{MHz}, \mathrm{CDCl}_{3}, 25^{\circ} \mathrm{C}\right): \delta 8.56$ (dd, $J=0.7$, 2.7 Hz, 2H, Pyz), 7.87 (s, 2H, Py), 7.76 (dd, $J=0.7,1.6$ Hz 2H, Pyz), 6.50 (dd, $J=1.6,2.7 \mathrm{~Hz} 2 \mathrm{H}, \mathrm{Pyz}), 4.86\left(\mathrm{~d}, J=5.4 \mathrm{~Hz} 2 \mathrm{H}, \mathrm{CH}_{2}\right), 2.51(\mathrm{t}, J=5.9$ $\mathrm{Hz} 1 \mathrm{H}, \mathrm{OH})$

dipyrazol-3-yl-4-formylpyridine (3). Dimethylsulfoxide $(1.3 \mathrm{ml}, 18.3$ mmol) in dichloromethane (15 ml) was added to oxalyl dichloride (727 $\mu$, $8.3 \mathrm{mmol})$ in dichloromethane $(35 \mathrm{ml})$ at $-78{ }^{\circ} \mathrm{C}$ and the mixture was stirred for 30 minutes. 2 (1.00 g, $4.15 \mathrm{mmol})$ in dichloromethane $(200 \mathrm{ml})$ was added drop wise over a period of 1 hour to the resulting mixture. After 
additional stirring for 1 hour, triethylamine $(5.82 \mathrm{ml}, 41.7 \mathrm{mmol})$ was added to give clear solution, and then allow the temperature increase to r.t. The resulting solution was washed with water and extracted with dichloromethane. The crude product was purified by silica-gel column chromatography using ethylacetate/dichloromethane (1 / 1, v / v) as an eluent to yield $824 \mathrm{mg}$ of 3 (3.44 mmol, 83 \%). Anal. Calcd for $\mathrm{C}_{12} \mathrm{H}_{9} \mathrm{~N}_{5} \mathrm{O}_{1}$ : C, 60.25; H, 3.79; N, 29.27. Found: C, 59.95; H, 3.97; N, 28.99\%. ${ }^{1} \mathrm{H}-\mathrm{NMR}\left(270 \mathrm{MHz}, \mathrm{CDCl}_{3}\right.$, $25^{\circ} \mathrm{C}$ ): $\delta 10.16$ (s, $1 \mathrm{H}, \mathrm{CHO}$ ), 8.57 (dd, $\left.J=0.7,2.6 \mathrm{~Hz} 2 \mathrm{H}, \mathrm{Pyz}\right), 8.27$ (s, 2H, Py), 7.81 (dd, $J=0.7,1.6$ Hz 2H, Pyz), 6.54 (dd, $J=1.6,2.6$ Hz 2H, Pyz).

2,6-di(pyrazol-1-yl)-4-(4,4,5,5-tetramethyl-1-oxido-3-ylooxy-4,5-dihydro3H-imidazol-2'-yl)pyridine (dppNN). $\quad N, N^{\prime}$-dihydroxy-2,3-diamino2,3-dimethylbutane (150 $\mathrm{mg}, 1.04 \mathrm{mmol})$ in benzene $(6 \mathrm{ml})$ was added to 3 (750 $\mathrm{mg}, 1.02 \mathrm{mmol})$ in benzene $(7 \mathrm{ml})$ and stirred for 2 weeks to obtain white precipitate. The resulting white solid was filtered and dissolved with chloroform (10 ml) and then Sodium periodate (215 mg, $1.00 \mathrm{mmol})$ in water (5 ml) was added. After stirring for 30 minetes, deep blue solution was obtained and the mixuture was washed with water and extracted with dichloromethane. The crude product was purified by silica-gel column 
chromatography using ethylacetate/dichloromethane (3/1, v / v) as an eluent to yield $90 \mathrm{mg}$ of dppNN (0.25 mmol, $42 \%)$. Anal. Calcd for $\mathrm{C}_{18} \mathrm{H}_{20} \mathrm{~N}_{7} \mathrm{O}_{2}$ : C, 59.01; H, 5.50; N, 26.76. Found: C, 59.21; H, 5.51; N, 26.96\%.

[Fe(dppNN $\left.)_{2}\right]\left(\mathbf{B F}_{4}\right)_{2} \cdot\left(\mathbf{C H}_{3} \mathbf{C O C H}_{3}\right)$ (1): A solution of $\mathrm{Fe}\left(\mathrm{BF}_{4}\right)_{2} \cdot 6 \mathrm{H}_{2} \mathrm{O}(25$ $\mathrm{mg}, 0.16 \mathrm{mmol}$ ) in degassed acetone $(5 \mathrm{ml})$ was added to the blue solution of dppNN (35 mg, $0.06 \mathrm{mmol}$ ) in acetone (5 ml) and stirred for $30 \mathrm{~min}$ to obtain dark red solution. Diffusion of diethylether to the resulting solution gave dark red crystals of 1. Anal. Calcd for $\mathrm{C}_{62} \mathrm{H}_{50} \mathrm{~B}_{2} \mathrm{~F}_{8} \mathrm{Fe}_{5} \mathrm{~N}_{10} \cdot 4 \mathrm{H}_{2} \mathrm{O}$ : C, 51.00; H, 4.00; N, 9.59. Found: C, 51.18; H, 3.91; N, 9.50.

\section{2-2. X-ray crystallography}

A Crystal of $\mathbf{1}$ was mounted on a glass capillary, and data were collected at $-70{ }^{\circ} \mathrm{C}$ (Bruker SMART APEX diffractometer coupled with a CCD area detector with graphite monochromated Mo-K $\alpha(\lambda=0.71073 \AA)$ radiation). The structure was solved by a direct method and expanded by using Fourier techniques using SHELXTL program. Crystal data of $\mathbf{1}$ at $-73^{\circ} \mathrm{C}: \mathrm{C}_{39} \mathrm{H}_{37} \mathrm{~B}_{2} \mathrm{~F}_{8} \mathrm{FeN}_{14} \mathrm{O}_{5}, M=1011.30$, monoclinic space group $P 2_{1}, a=$ 
8.387(2), $b=23.039(4), c=12.215(2) \AA, \beta=96.587(3)^{\circ}, V=2346.4(7) \AA^{3}, Z$

$=2$. A total of 10549 were collected $\left(3^{\circ}<2 \theta<47^{\circ}\right)$ of which 5439 unique

reflections $(R($ int $)=0.0379)$ were measured. Residual $R$ and $w R$ were 0.0739 and 0.1941 , respectively, from the refinement on $F^{2}$ with $\mathrm{I}>2 \sigma(\mathrm{I})$; Empirical absorption corrections by SADABS (G. M. Sheldrick, 1994) were carried out. In the structure analyses, non-hydrogen atoms were refined with anisotropic thermal parameters. Hydrogen atoms were included in calculated positions and refined with isotropic thermal parameters riding on those of the parent atoms. Crystallographic data reported in this paper have been deposited with Cambridge Crystallographic Data Centre as supplementary publication no. CCDC 278362. These data can be obtained free of charge via www.ccdc.cam.ac.uk/conts/retrieving.html (or from the Cambridge Crystallographic Data Centre, 12, Union Road, Cambridge CB2 1EZ, UK; fas: (+44) 1223-336-033; or deposit @ccdc.cam.ac.uk).

\section{2-2. magnetic measurements}

DC magnetic susceptibility data of $\mathbf{1}$ were collected by using a 
Quantum Design MPMS-XL SQUID magnetometer at temperatures ranging from 5 to $340 \mathrm{~K}$. For the LIESST experiment, Xe lamp was used as light source and 500-600 nm light was isolated by using colour filter and was guided via an optical fiber into the SQUID magnetometer. Irradiation was performed inside the SQUID sample room at $5 \mathrm{~K}$.

\section{Results and Discussion}

\section{3-1. Syntheses of dppNN and $\left[\mathrm{Fe}\left(\mathrm{dppNN}_{2}\right)_{2}\right]\left(\mathrm{BF}_{4}\right)_{2} \cdot\left(\mathrm{CH}_{3} \mathrm{COCH}_{3}\right)(\mathbf{1})$}

A tridentate ligand of dppNN was synthesized from ethyl 2,6-di(pyrazol-1-yl)isonicotinate ${ }^{7}$ by three-step reactions. In the first step, the carboxyethyl group was reduced by sodium borohydrate affording a hydroxymethyl group, and the hydroxymethyl group was oxidized to a formyl group by the Swern oxidation in the second step. A reaction of formyl compound with $N, N^{\prime}$-dihydroxy-2,3-diamino-2,3-dimethylbutane followed by an oxidation with sodium periodate yielded the ligand dppNN in a moderate yield. An Iron(II) complex of $\left[\mathrm{Fe}(\mathrm{dppNN})_{2}\right]\left(\mathrm{BF}_{4}\right)_{2} \cdot\left(\mathrm{CH}_{3} \mathrm{COCH}_{3}\right)(\mathbf{1})$ was obtained by a reaction of dppNN 
with $\mathrm{Fe}\left(\mathrm{BF}_{4}\right)_{2} \cdot 4 \mathrm{H}_{2} \mathrm{O}$. All compounds were characterized by elemental analyses.

\section{3-1. Descriptions of Crystal structures: 1}

X-ray crystal structure analysis for $\mathbf{1}$ was performed at $200 \mathrm{~K}$, and ORTEP diagram of the complex molecule was depicted in Figure 1. 1 crystallized in monoclinic space group $P 2_{1}$, and the asymmetric unit is composed of one complex cation, two tetrafluoroborate anions, and one acetone molecule. A central iron(II) ion is coordinated by six nitrogen atoms from two tridentate dppNN giving distorted octahedral coordination geometry. Coordination bond lengths of Fe-N1 and Fe-N8 (N1 and N8 are pyridyl nitrogen atoms) are 1.889(6) $\AA$ and 1.903(6) $\AA$, respectively, which are shorter than the coordination bond lengths of $\mathrm{Fe}^{\mathrm{II}}$ ion with pyrazole nitrongen atoms (Fe-N2, N4, N9 and N11: 1.958(6) - 1.983(7) $\AA$ ). The average coordination bond length of $1.948 \AA$ and bite angles (N1-Fe-N2, -N5, N8-Fe-N9, -N12) of 79.7(3) - 80.4(3) are typical values for low spin iron(II) ions in the series of this tridentate ligand.[9] Interatomic distances of $\mathrm{N}-\mathrm{O}$ groups in nitronyl nitroxides are in the range of $1.26(1)-1.28(1) \AA$ for N6-O1, N7-O2, N13-O3 and N14-O4, respectively, which are characteristic 
of radical centers. It should be mentioned that no significant intermolecular contact between the NO groups of dppNN ( $>4 \AA$ ) was observed and cations are magnetically isolated.

\section{3-3. Magnetic susceptibility measurements of 1}

Magnetic susceptibility measurements for $\mathbf{1}$ was done in the temperature range of $5-340 \mathrm{~K}$ and a $\chi_{\mathrm{m}} T-T$ plot was shown in Figure 2 . The $\chi_{\mathrm{m}} T$ values below $130 \mathrm{~K}$ are almost constant $\left(0.83\right.$ emu $\left.\mathrm{mol}^{-1} \mathrm{~K}\right)$, which corresponds to the uncorrelated two radical spins. It is pointed out that coordination bond lengths at $200 \mathrm{~K}$ are typical values for low-spin iron(II) ions, which magnetically isolate two radicals in the molecule. Sudden decrease of the $\chi_{\mathrm{m}} T$ values below $30 \mathrm{~K}$ is due to weak intermolecular antiferromagnetic interactions between dppNN radicals. As the temperature was increased up to $340 \mathrm{~K}$, gradual increase of $\chi_{\mathrm{m}} T$ values was observed and this increase was recovered in the cooling process, suggesting this phenomenon being a reversible thermal spin-crossover phenomenon. $\mathrm{A} \chi_{\mathrm{m}} T$ value of 1.21 emu mol${ }^{-1} \mathrm{~K}$ at $340 \mathrm{~K}$ corresponds to $14 \%$ of the central iron(II) ion being in the high spin state. The sum of Curie constants for isolated 
iron(II) ion and two radicals is $3.75 \mathrm{emu} \mathrm{mol}^{-1} \mathrm{~K}$, and the critical temperature of the spin transition is higher than $340 \mathrm{~K}$, where. When the sample was irradiated with $500-600 \mathrm{~nm}$ light at $5 \mathrm{~K}$, the $\chi_{\mathrm{m}} T$ values of 1 abruptly increased, suggesting that the metastable HS state was trapped. The $\chi_{\mathrm{m}} T$ values of the light irradiated sample (metastable high-spin state) increased as the temperature was increased, reaching a maximum value of $1.27 \mathrm{emu} \mathrm{mol}^{-1}$ $\mathrm{K}$ at $26 \mathrm{~K}$. The $\chi_{\mathrm{m}} T$ value is smaller than the value $\left(3.75 \mathrm{emu} \mathrm{mol}^{-1} \mathrm{~K}\right.$ ) expected for the isolated iron(II) ion and two radicals, and this might be due to the incomplete spin conversion or antiferromagnetic interactions between high spin iron(II) ion and organic radicals. Further increase of temperature resulted in sudden decrease of $\chi_{\mathrm{m}} T$ values, which indicates the occurrence of complete relaxation from the metastable HS state to the LS state.

\section{Conclusion}

We prepared a novel iron(II) complex with nitronyl nitroxide radicals, and its structure and magnetic property were studied. Magnetic susceptibility measurement suggests central iron(II) ion show the thermal spin transition with a critical temperature higher than $340 \mathrm{~K}$, and LIESST 
phenomenon was confirmed by light irradiation at $5 \mathrm{~K}$.

\section{Ackowledgement}

This work was partially supported by a Grant-in-aid for Scientific

Researches from the Ministry of Education, Culture, Sports, Science and Technology, Japan, and by the COE and TARA projects of University of Tsukuaba.

\section{References}

[1] (a) M. Kimura, T. Horai, T. Muto, K. Hanabusa, H. Shirai, Chem. Lett. (1999) 1129; (b) S. Kelch, M. Rehahn, Macromolecules 32 (1999) 5818. (c) U. Velten, B. Lahn, M. Rehahn, Macromol. Chem. Phys. 198 (1997) 2789; (d) U. S. Schubert, C. Eschbaumer, Angew. Chem. Int. Ed. 41 (2002) 2892.

[2] U. S. Schubert, C. H. Weidl, C. N. Moorefield, G. R. Baker, G. R. Newkome, Polym. Preprints 40 (1999) 940.

[3] B. J. Holliday, C. A. Mirkin, Angew. Chem. Int. Ed. 40 (2001) 2022. 
[4] (a) M. Kinoshita, P. Turek, M. Tamura, K. Nozawa, D. Shiomi, Y. Nakazawa, M. Ichikawa, M. Takahashi K. Awaga, T. Inabe, and Y. Maruyama, Chem. Lett. (1991) 1225; (b) M. Takahashi, P. Turek, Y. Nakawaza, M. Tamura, K. Nozawa, D. Shiomi, M. Ishikawa, and M. M. Kinoishita, Phys. Rev. Lett. 67 (1991) 746; (c) T. Sugawara, M. Matsushita, A. Izuoka, N. Wada, N. Takeda, and M. Ishikawa, J. Chem. Soc., Chem. Commun. (1994) 1723; (d) M. M. Matsushita, A. Izuoka, T. Sugawara, T. Kobayashi, N. Wada, N. Takeda, and M. Ishikawa, J. Am. Chem. Soc. 119 (1997) 4369.

[5] (a) A. Caneschi, D. Gateschi, R. Sessoli, and P. Rey, Acc. Chem. Res. 22 (1989) 392; (b) M. Fettouhi, M. Khaled, A. Waheed, S. Golhen, L. Ouhab, J. P. Sutter, and O. Kahn, Inorg. Chem. 38 (1999) 3967; （c) A. Caneschi, F. Ferrao, D. Gateschi, P. Rey, and R. Sessoli, Inorg. Chem. 29 (1990) 4217; (d) H. Iwamura, K. Inoue, and T. Hayamizu, Pure Appl. Chem. 68 (1996) 243.

[6] (a) A. Iino, T. Suzuki, S. Kaizaki, J. Chem. Soc., Dalton. Trans. (2003) 4604; (b) J. R. Gardinier, R. Clérac, F. P. Gabbaï, J. Chem. Soc., Dalton. Trans. (2001) 3453; (c) Z. Liu, Z. Lu, D. Zhang, Z. Jiang, L. 
Li, C. Liu, D. Zhu, Inorg. Chem. 43 ()2004 6620; (d) M. Fettouhi, B.

E. Ali, A. M. EI-Ghanam, S. Golhen, L. Ouahab, N. Daro, J.-P. Sutter, Inorg. Chem. 41 (2002) 3705; (e) H. Oshio, T. Watanabe, A. Ohto, T. Ito, T. Ikoma, S. Tero-Kubota, Inorg. Chem. 36 (1997) 3014.

[7] C. Stroh, P. Turek, P. Rabu, R. Ziessel, Inorg. Chem. 40 (2001) 5334.

[8] (a) E. Fursova, G. Romanenko, V. Ikorskii, V. Ovcharenko, polyhedron 22 (2003) 1957. (b) E. Fursova, V. Ovcharenko, G. Romanenko, E. V. Tretyakov, Tetrahedron Lett. 44 (2003) 6397. (c) S. Fokin, V. Ovcharenko, G. Romanenko, V. Ikorskii, Inorg. Chem. 43 (2004) 969. (d) E. V. Tretyakov, S. Fokin, G. Romanenko, V. Ovcharenko, polyhedron, 22 (2003) 1965. (e) K. Fegy, N. Sanz, D. Luneau, E. Belorizki, P. Rey, Inorg. Chem. 37 (1998) 4518. (f) C. Lescop, E. Belorizky, D. Luneau, P. Rey, Inorg. Chem. 41 (2002) 3375. (g) C. Aoki, I. Ishida, T. Nogami, Inorg. Chem. 42 (2003) 7616. (h) T. Yeltov, V. Ovcharenko, V. Ikorskii, G. Romanenko, S. Vasilevsky, polyhedron, 20 (2001) 1215. (i) H. Mori, O. Nagano, M. Kozaki, D. Shimoi, K. Sato, T. Takui,K. Okada, Polyhedron, 20 (2001) 1663.

[9] A. Caneschi, D. Gateschi, N. Lalioti, C. Sangregorio, R. Sessoli, G. 
Venturi, A. Vindigni, A. Rettori, M. G. Pini, and M. A. Novak, Angew. Chem., Int. Ed. 40 (2001) 1760.

[10] (a) V. A. Money, I. R. Evans, M. A. Halcrow, A. E. Goeta, and J. A. K. Howard, Chem. Commun. (2003) 158; (b) J. M. Holland, J. A. McAllister, Z. Lu, C. A. Kilner, M. T.-Pett, and M. A. Halcrow, Chem. Commun. (2001) 577; c) V. A. Money, J. Elhaïk, M. A. Halcrow, and J. A. K. Howard, J. Chem. Soc., Dalton. Trans. (2004) 1516; (d) V. A. Money, J. Elhaïk, I. R. Evans, M. A. Halcrow, and J. A. K. Howard, J. Chem. Soc., Dalton. Trans. (2004) 65; (e) V. A. Money, J. Elhaïk, S. A. Barrett, C. A. Kilner, I. R. Evans, and M. A. Halcrow, J. Chem. Soc., Dalton. Trans. (2003) 2053;

[11] T. Vermonden, D. Branowska, A. T. M. Marcelis, and E. J. R. Sudhölter, Tetrahedron 59 (2003) 5039.

[12] E. F. Ullman, J. H. Osiecki, D. G. B. Boocock, R. Darcy, J. Am. Chem. Soc. 94 (1972) 7049. 


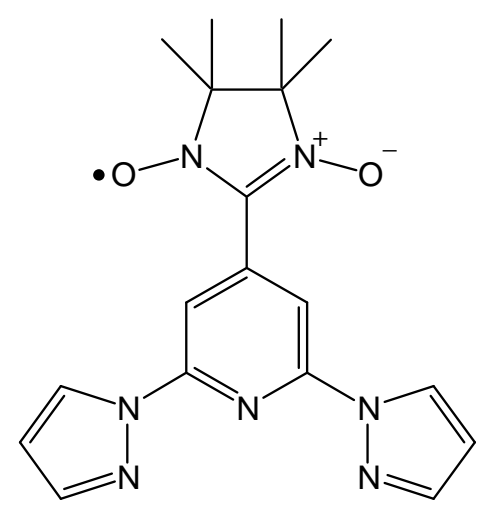

Scheme 1. Molecular structure of dppNN. 
Figure captions

Figure 1. ORTEP diagram of $\mathbf{1}$. Selected interatomic distances $(\AA)$ for $\mathbf{1}$ : Fe1-N1 1.889(6), Fe1-N2 1.974(6), Fe1-N5 1.958(6), Fe1-N8 1.903(6), Fe1-N9 1.983(7), Fe-N12 1.982(8), O1-N6 1.28(1), O2-N7 1.27(1), O3-N13 1.26(1), O4-N14 1.27(1).

Figure 2. $\chi_{\mathrm{m}} T-T$ plot for $\mathbf{1}$ 


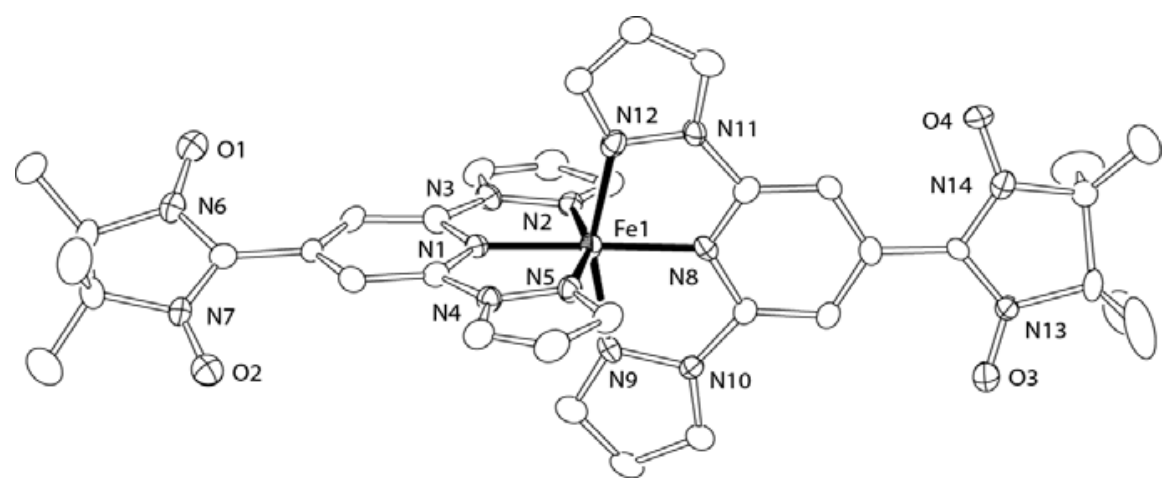

Figure 1. ORTEP diagram of $\mathbf{1}^{2+}$. Selected interatomic distances $(\AA)$ for $1^{2+}$ : Fe1-N1 1.889(6), Fe1-N2 1.974(6), Fe1-N5 1.958(6), Fe1-N8 1.903(6), Fe1-N9 1.983(7), Fe-N12 1.982(8), O1-N6 1.28(1), O2-N7 1.27(1), O3-N13 1.26(1), O4-N14 1.27(1). 


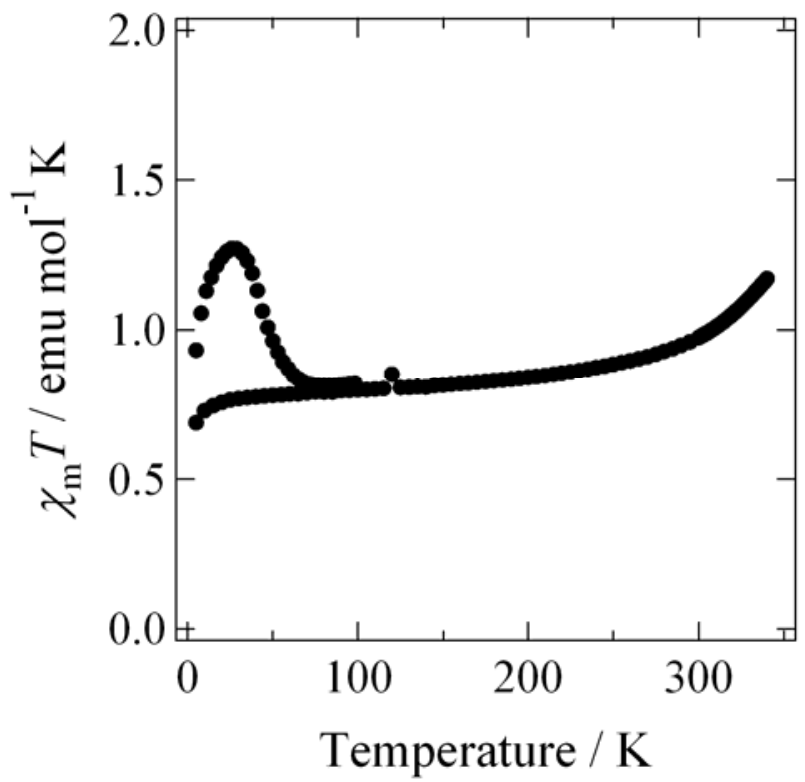

Figure 2. $\chi_{\mathrm{m}} T-T$ plot for $\mathbf{1}\left(\mathrm{BF}_{4}\right)_{2} \cdot\left(\mathrm{CH}_{3} \mathrm{COCH}_{3}\right)$. 
Graphical abstract

An iron(II) complex with nitronyl nitroxide radicals was synthesized and structure and magnetic properties were presented. The central iron(II) ion was coordinated by two tridentate ligands with nitroniyl nitroxide radicals. In a magnetic susceptibility measurement, $\mathbf{1}\left(\mathrm{BF}_{4}\right)_{2} \cdot\left(\mathrm{CH}_{3} \mathrm{COCH}_{3}\right)$ showed thermally and light induced spin transitions.

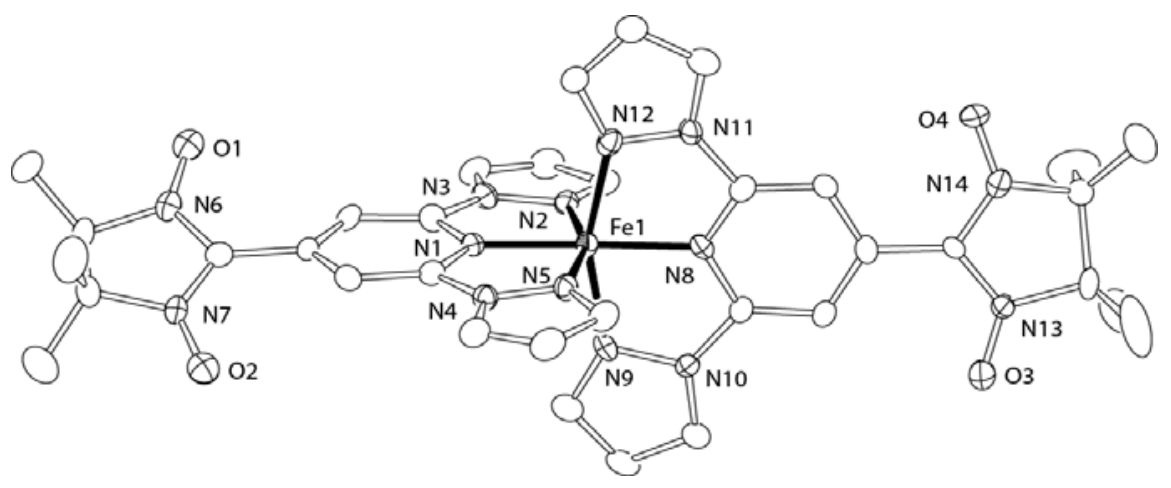

